\title{
Enzymologic and Pharmacologic Profile of Loxoprofen Sodium and Its Metabolites
}

\author{
Masahiro Noguchi, ${ }^{*, a}$ Aishi KImoto, ${ }^{a}$ James Kevin Gierse, ${ }^{b}$ Mark Crossfield WalKer, ${ }^{b}$ \\ Ben Scott ZweIFEL, ${ }^{b}$ Kazutoshi NozAKI, ${ }^{a}$ and Masao SASAMATA ${ }^{a}$ \\ ${ }^{a}$ Pharmacology Research Laboratories, Drug Discovery Research, Astellas Pharma Inc.; 21 Miyukigaoka, Tsukuba, \\ Ibaraki 305-8585, Japan: and ${ }^{b}$ Pfizer Global Research and Development; 700 Chesterfield Parkway West, Chesterfield, \\ Missouri, 63017, U.S.A. Received April 28, 2005; accepted August 20, 2005; published online August 23, 2005
}

We investigated the mechanism of inhibition of loxoprofen sodium, a non-steroidal anti-inflammatory drug (NSAID), and its active metabolite (loxoprofen-SRS) on cyclooxygenase (COX). In in vitro assays, loxoprofen sodium appeared inactive against recombinant human COX-1 and COX-2, whereas loxoprofen-SRS inhibited both. In the investigation of kinetic behavior, loxoprofen-SRS showed time-dependent inhibition for both isozymes. Human whole blood assay also showed that loxoprofen-SRS possesses the profile of a non-selective inhibitor for COX. In a rat air pouch model, oral administration of loxoprofen sodium lowered prostaglandin (PG) $E_{2}$ in both fluid exudates of the inflammatory pouch and stomach tissue with $E_{50}$ values of 2.0 and $2.1 \mathrm{mg} / \mathrm{kg}$, respectively. Additionally, platelet thromboxane $B_{2}$ production was also inhibited by loxoprofen sodium $\left(\mathbf{E D}_{50}\right.$ of $0.34 \mathrm{mg} / \mathrm{kg}$ ). In a rat carrageenan-induced paw edema model, loxoprofen sodium dose-dependently reduced the paw edema, accompanied by a decrease in $\mathrm{PGE}_{2}$ content in inflamed paw exudates. These findings suggest that the COX inhibitory activity of loxoprofen sodium is attributable to its active metabolite, loxoprofen-SRS, and that loxoprofen-SRS shows non-selective inhibition for COX.

Key words loxoprofen sodium; cyclooxygenase; prostaglandin; non-steroidal anti-inflammatory drug (NSAID)

Cyclooxygenase (COX), the central enzyme in prostanoid biosynthesis, catalyzes the conversion of arachidonic acid to prostaglandin (PG) $\mathrm{H}_{2}$. The enzyme encompasses two distinct enzymatic activities: a cyclooxygenase function, which converts arachidonic acid to $\mathrm{PGG}_{2}$, and a peroxidase function, which transforms $\mathrm{PGG}_{2}$ to $\mathrm{PGH}_{2} \cdot{ }^{1)} \mathrm{PGH}_{2}$ is then converted to biologically active $\mathrm{PGs}$ such as $\mathrm{PGE}_{2}$ by tissue-specific isomerases such as $\mathrm{PGE}_{2}$ synthase. ${ }^{2)}$ Two isoforms of COX have been identified, COX-1 and COX-2. The tissue distribution of COX-1 and COX-2 and their respective physiological roles have been extensively reviewed. ${ }^{3,4)}$

Nonsteroidal anti-inflammatory drugs (NSAIDs) are widely used for the treatment of conditions characterized by pain or inflammation such as rheumatoid arthritis and osteoarthritis. Conventional NSAIDs inhibit both COX-1 and COX-2. ${ }^{4,5)}$ The therapeutic effects of NSAIDs are thought to result from the inhibition of COX-2, which reduces the formation of PGs and provides the desired anti-inflammatory activity. ${ }^{6,7)}$ However, their lack of selectivity for COX is associated with adverse events such as gastrointestinal injury and inhibition of platelet function, presumably as a result of the additional inhibition of COX-1. ${ }^{7)}$ Loxoprofen sodium (Fig. 1A), a phenylpropionate NSAID that is converted into an active metabolite (loxoprofen-SRS) (Fig. 1B) in vivo, ${ }^{8)}$ is commonly used for the treatment of pain and inflammation in Japan, and exhibits relatively weak gastrointestinal ulcerogenicity. ${ }^{9)}$ The COX selectivity of many NSAIDs has been investigated using various in vitro and in vivo bioassays, ${ }^{10,11)}$ but those of loxoprofen sodium and loxoprofen-SRS have not been investigated in detail.

In the present study, we investigated the COX selectivity of these compounds using established in vitro and in vivo bioassays.

\section{MATERIALS AND METHOD}

Materials Arachidonic acid was obtained as the sodium salt from Nu-Chek Prep (Elysian, MN, U.S.A.). Recombinant human COX-1 and COX-2 were purified as previously described. ${ }^{5)}$ Loxoprofen sodium $2 \mathrm{H}_{2} \mathrm{O}$ (Shiono Chemical, Tokyo, Japan) and Loxoprofen-SRS (Astellas Pharma Inc., Tokyo, Japan) were synthesized. Phenol, $N, N, N^{\prime}, N^{\prime}$-tetramethyl-p-phenylenediamine (TMPD), haemin chloride, CHAPS, EDTA, Tris and all standard buffers, calcium ionophore A-23187 and Lambda carrageenan were obtained from Sigma Chemical Co. (St. Louis, MO, U.S.A.). Lipopolysaccharide (LPS) was purchased from Difco Laboratories (Detroit, MI, U.S.A.).

(A) Loxoprofen sodium

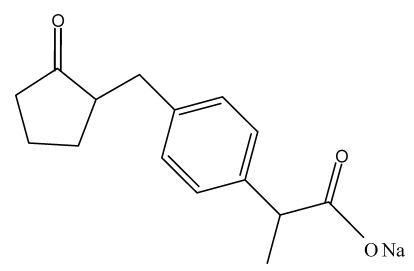

(B) Loxoprofen-SRS

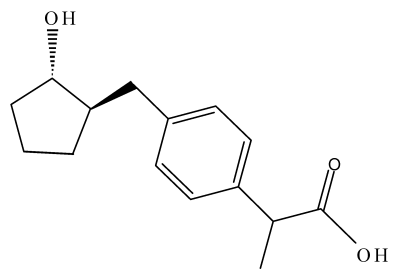

Fig. 1. Structures of (A) Loxoprofen Sodium and (B) Loxoprofen-SRS 
PGE $_{2}$ ELISA Expression of COX protein in $\mathrm{Sf} 9$ insect cells was determined by assessing PG-synthesis capability in homogenates from cells incubated for $3 \mathrm{~d}$ with $\mathrm{COX}-1$ or COX-2 baculovirus. Cells expressing COX-1 or COX-2 were homogenized as previously described, ${ }^{5)}$ incubated with arachidonic acid $(10 \mu \mathrm{M})$, and COX activity was determined by monitoring $\mathrm{PGE}_{2}$ production. No COX activity was detected in mock-infected Sf9 cells. Loxoprofen sodium and Loxoprofen-SRS were dissolved in ethanol to make stock solutions of $10 \mathrm{~mm}$, which were diluted into a potassium phosphate buffer $(50 \mathrm{~mm}, \mathrm{pH} 8.0)$ to final concentrations of 2.5 $2500 \mu \mathrm{M}$ and $0.1-100 \mu \mathrm{M}$, respectively, followed by the addition of crude $1 \%$ CHAPS homogenates $(2-10 \mu \mathrm{g}$ protein). After 20-min of pre-incubation, arachidonic acid $(10 \mu \mathrm{M})$ was added to the enzyme-inhibitor mixture, and COX activity was assessed by the amount of $\mathrm{PGE}_{2}$ formed during 20min incubation with substrate. $\mathrm{PGE}_{2}$ was determined by ELISA (Cayman Chemical, Ann Arbor, MI, U.S.A.). During the incubation approximately $150 \mathrm{ng} / \mathrm{ml}$ of $\mathrm{PGE}_{2}$ were produced.

Oxygen Consumption Assay Oxygen consumption was measured directly with a Clark-style polarographic electrode in a $600 \mu \mathrm{l}$ reaction vessel (Instech, Model 203; Horsham, PA, U.S.A.). The assay mixture contained $100 \mathrm{~mm}$ Tris-HCl, $\mathrm{pH}$ 8.0, $1 \mu \mathrm{m}$ haem, $500 \mu \mathrm{m}$ phenol, $100 \mu \mathrm{m}$ arachidonic acid and $0-20 \mu \mathrm{g}$ of protein. The assay was initiated by the addition of arachidonate, and rate measurements were taken in the linear portion of the assay (first $10-15 \mathrm{~s}$ ). For $\mathrm{IC}_{50}$ determinations, loxoprofen sodium or loxoprofen-SRS were incubated with $0-20 \mu \mathrm{g}$ of protein COX-1 or COX-2 for $1 \mathrm{~min}$ prior to the addition of arachidonic acid.

For time-dependent mechanism of action studies, $100 \mathrm{~mm}$ Tris- $\mathrm{HCl}, \mathrm{pH}$ 8.0, $1 \mu \mathrm{m}$ haem (bovine haemin chrolide in dimethyl sulfoxide), $500 \mu \mathrm{M}$ phenol, $100 \mu \mathrm{M}$ of arachidonic acid and several concentrations of loxoprofen-SRS were preincubated from 0 to $300 \mathrm{~s}$ prior to the addition of arachidonic acid.

Peroxidase Assay COX activity was indirectly measured by utilizing TMPD as a co-substrate with arachidonic acid. TMPD does not react without the presence of a hydroperoxide substrate. COX-1 or COX-2 $(0-5 \mu \mathrm{g}$ of protein) and several concentrations of loxoprofen sodium or loxoprofenSRS were combined with $100 \mathrm{~mm}$ Tris- $\mathrm{HCl}, \mathrm{pH} 8.1,170 \mu \mathrm{M}$ TMPD, $1 \mu \mathrm{M}$ haem and $100 \mu \mathrm{M}$ arachidonic acid. OD at 590 $\mathrm{nm}$ was measured after $5 \mathrm{~min}$ at room temperature. One unit was defined as the amount of enzyme necessary to generate a change of 1 OD at $590 \mathrm{~nm}$ during 5-min incubation.

Human Whole Blood Assay To evaluate the effect of loxoprofen-SRS on COX-1-mediated thromboxane (TX) $\mathrm{B}_{2}$ production, venous blood was collected from healthy human donors using heparin as an anti-coagulant $(n=5)$. Blood samples $(0.2 \mathrm{ml}$ of a $1: 4$ dilution in RPMI 1640 medium $)$ were incubated in 96 -well culture plates for $15 \mathrm{~min}$ at $37^{\circ} \mathrm{C}$ with loxoprofen-SRS dissolved in ethanol (final concentration $<1 \%)$. Loxoprofen-SRS $(0.001-200 \mu \mathrm{M})$ was examined in duplicate. Calcium ionophore A-23187 (final concentration $20 \mu \mathrm{g} / \mathrm{ml}$ ) was used to stimulate $\mathrm{TXB}_{2}$ production by incubation with blood for $10 \mathrm{~min}$ at $37^{\circ} \mathrm{C}$. The reaction was stopped by placing the samples on ice. The samples were then centrifuged at $800 \times \boldsymbol{g}$ for $10 \mathrm{~min}$ at $4{ }^{\circ} \mathrm{C}$ and the supernatants were collected for $\mathrm{TXB}_{2}$ analysis by ELISA. $\mathrm{TXB}_{2}$ levels produced were approximately $10 \mathrm{ng} / \mathrm{ml}$. To evaluate the effect of loxoprofen-SRS on COX-2-mediated $\mathrm{PGE}_{2}$ production, undiluted blood samples $(n=5)(0.2 \mathrm{ml})$ were incubated in 96-well culture plates for $24 \mathrm{~h}$ at $37^{\circ} \mathrm{C}$ with LPS $(10 \mu \mathrm{g} / \mathrm{ml})$ and loxoprofen-SRS $(0.001-200 \mu \mathrm{M})$ dissolved in ethanol at a final vehicle concentration of $<1 \%$. The reaction was stopped by cold centrifugation at $800 \times \boldsymbol{g}$ for $10 \mathrm{~min}$ at $4{ }^{\circ} \mathrm{C}$ to pellet the cells, and the supernatants were recovered for quantitation of $\mathrm{PGE}_{2}$ by ELISA.

Air-Pouch Model of Inflammation Male Lewis rats (Charles River Laboratories, Wilmington, MA, U.S.A.) weighing $175-200 \mathrm{~g}$ were used in groups of 6 . Air pouches were produced by s.c. injection of $20 \mathrm{ml}$ of sterile air into the interscapular area of the back. Animals were fasted with free access to water for 16 to $24 \mathrm{~h}$ prior to drug administration. After $24 \mathrm{~h}, 2 \mathrm{ml}$ of a $1 \%$ solution of carrageenan dissolved in saline was injected directly into the pouch to produce an inflammatory response. Loxoprofen sodium $(0.001-200$ $\mathrm{mg} / \mathrm{kg}$ ) was administrated orally $1 \mathrm{~h}$ prior to carrageenan injection. At $3 \mathrm{~h}$ after carrageenan injection, the pouch fluid was washed with $1 \%$ heparin/physiological saline solution. The fluid was centrifuged at $800 \times \boldsymbol{g}$ for $10 \mathrm{~min}$ at $4{ }^{\circ} \mathrm{C}$, and the supernatants were collected for $\mathrm{PGE}_{2}$ analysis. $\mathrm{PGE}_{2}$ levels were quantitated by ELISA. Blood was also obtained in the same manner and platelet $\mathrm{TXB}_{2}$ was measured by ELISA. The stomachs of these animals were excised, opened and cleaned, and the mucosal lining was dissected out and frozen in liquid nitrogen. Stomach tissue was processed by homogenization in $70 \%$ ethanol followed by centrifugation. The supernatants were then dried under a stream of nitrogen. The samples were resuspended in ELISA buffer and the $\mathrm{PGE}_{2}$ were quantified by ELISA. Basal levels of $\mathrm{PGE}_{2}$ produced were approximately $2.4 \mathrm{ng} / \mathrm{ml}$, and the stimulated levels were $10-20 \mathrm{ng} / \mathrm{ml}$.

Measurement of Edema and PG Production in Carrageenan-Injected Rat Paws Male Sprague-Dawley rats (Charles River Laboratories, Wilmington, MA, U.S.A.) weighing $180-205 \mathrm{~g}$ were used in groups of 8 . Rats were fasted for $16 \mathrm{~h}$, then given loxoprofen sodium $(0.3-30 \mathrm{mg} /$ $\mathrm{kg}$ ) by oral administration. Two hours after administration, paw edema was produced by injecting $50 \mu \mathrm{l}$ of $1 \%$ carrageenan in saline into the left hind paw. Three hours later, paw volume was measured by water displacement using a plethysmometer (UGO Basil, Comerio, Italy). The rats were then euthanized with diethyl ether, and the left hind paw was removed and injected with $0.1 \mathrm{ml}$ of $10 \mu \mathrm{M}$ indomethacin in saline to prevent further production of eicosanoids. The paws were then lacerated with a scalpel, suspended off the bottom of a polypropylene centrifuge tube with an Eppendorf pipette tip and centrifuged $\left(2000 \times \boldsymbol{g}, 15 \mathrm{~min}, 4^{\circ} \mathrm{C}\right)$ to obtain inflammatory exudate. $\mathrm{PGE}_{2}$ levels in the paw exudate were determined by ELISA.

Statistics The $\mathrm{IC}_{50}$ value for $\mathrm{PGE}_{2}$ production was defined as the concentration of inhibitor that resulted in onehalf the maximal response, calculated by a two-parameter $\log$ fit. $\mathrm{IC}_{50}$ values determined in the oxygen consumption and peroxidase assay were defined as the concentration of inhibitor that inhibited one-half of the difference between maximal and minimal response, calculated by four-parameter logfit. For the human whole blood assay and the rat air pouch model, the $\mathrm{IC}_{50}$ value and $\mathrm{ED}_{50}$ values were estimated from a 
(A)

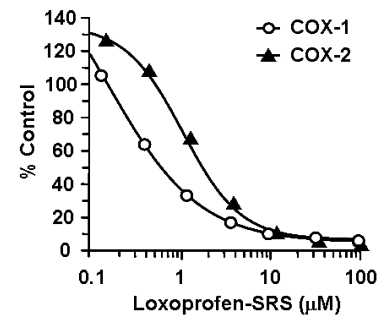

(B)

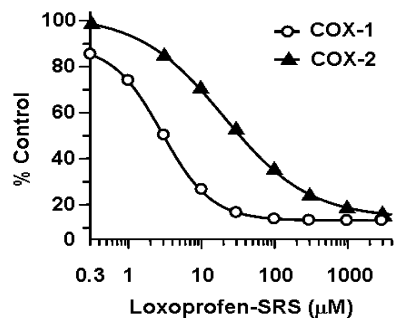

(C)
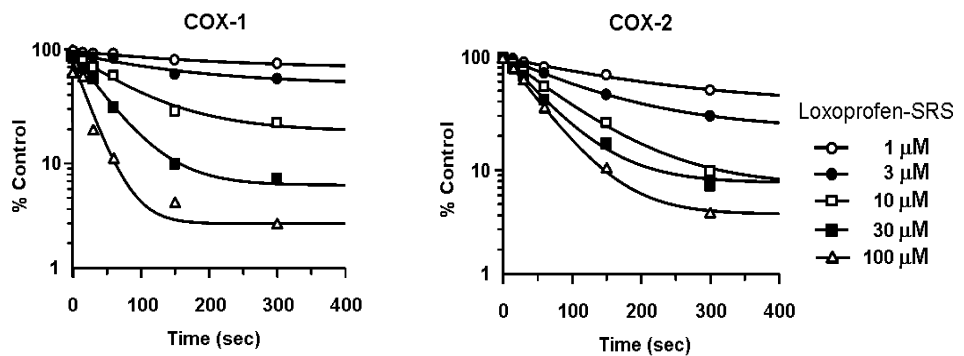

Fig. 2. Inhibitory Effects of Loxoprofen-SRS on Human COX-1 and COX-2

(A) $\mathrm{PGE}_{2}$ ELISA: $\mathrm{PGE}_{2}$ was measured after a $20 \mathrm{~min}$ incubation with $10 \mu \mathrm{M}$ arachidonic acid following a 20 min pre-incubation with loxoprofen-SRS. (B) Oxygen consumption assay: maximal rate of oxygen consumption was measured upon addition of $100 \mu \mathrm{M}$ arachidonic acid, after 1 min pre-incubation of loxoprofen-SRS with enzyme. (C) Time-dependent inhibition of COX-1 and COX-2 measured using the oxygen consumption assay: maximal rate of oxygen consumption was measured upon addition of $100 \mu \mathrm{M}$ arachidonic acid, after $0-300$ s pre-incubation of loxoprofen-SRS with enzyme.

four-parameter logistic model with fixed two parameters, namely the minimum ( $0 \%$ inhibition) and maximum $(100 \%$ inhibition). In the carrageenan-induced paw edema model, statistical significance was determined by Student's $t$-test or Dunnett's multiple range test. A $p$ value of $<0.05$ was considered significant.

\section{RESULTS}

Inhibition of COX Activity in Vitro In the $\mathrm{PGE}_{2}$ ELISA, oxygen consumption, and peroxidase assays, loxoprofen sodium had hardly any inhibitory effect on either COX-1 or COX-2 (Table 1). In contrast, loxoprofen-SRS dose-dependently inhibited both recombinant human COX-1 and COX-2 in all three in vitro assays. When either COX-1 or COX-2 was incubated with arachidonic acid and then assayed for $\mathrm{PGE}_{2}$ production under pre-incubation conditions, it was found that loxoprofen-SRS dose-dependently inhibited both forms of the enzyme. The $\mathrm{IC}_{50}$ values for COX-1 and COX-2 were $0.64 \mu \mathrm{M}$ and $1.85 \mu \mathrm{M}$, respectively (Table 1 , Fig. 2A). The inhibition of COX activity of loxoprofen-SRS was confirmed when COX activity was measured directly using the oxygen consumption method. COX-1 or COX-2 (0$20 \mu \mathrm{g}$ of protein) was preincubated for $1 \mathrm{~min}$ with loxoprofen-SRS $(0.3-3000 \mu \mathrm{M})$ followed by the addition of $100 \mu \mathrm{M}$ arachidonic acid. The $\mathrm{IC}_{50}$ values for COX-1 and COX-2 were $2.9 \mu \mathrm{M}$ and $20.3 \mu \mathrm{M}$, respectively (Table 1, Fig. 2B). Additionally, the COX inhibition activity was confirmed using the peroxidase assay, an $\mathrm{IC}_{50}$ values for COX-1 and COX-2 were $11.2 \mu \mathrm{M}$ and $106.8 \mu \mathrm{M}$, respectively (Table 1 ). The COX-2 selectivity of loxoprofen-SRS, namely, the COX-1 $\mathrm{IC}_{50} / \mathrm{COX}-2 \mathrm{IC}_{50}$ ratio, was $0.14-0.35$ (Table 1 ). In the timedependent mechanism of action studies conducted using the oxygen consumption assay, loxoprofen-SRS demonstrated
Table 1. COX Inhibition of Loxoprofen Sodium and Loxoprofen-SRS in Three in Vitro Assays

\begin{tabular}{|c|c|c|c|c|c|}
\hline & \multicolumn{2}{|c|}{$\begin{array}{l}\text { Loxoprofen sodium } \\
\qquad \mathrm{IC}_{50}(\mu \mathrm{M})\end{array}$} & \multicolumn{3}{|c|}{$\begin{array}{l}\text { Loxoprofen-SRS } \\
\mathrm{IC}_{50}(\mu \mathrm{M})\end{array}$} \\
\hline & COX-1 & COX-2 & $\mathrm{COX}-1$ & COX-2 & $\begin{array}{c}\text { COX-2 } \\
\text { selectivity }^{a)}\end{array}$ \\
\hline $\mathrm{PGE}_{2}$ ELISA & $>2500$ & $>2500$ & 0.64 & 1.85 & 0.35 \\
\hline $\begin{array}{l}\text { Oxygen } \\
\text { consumption }\end{array}$ & ND & ND & 2.9 & 20.3 & 0.14 \\
\hline Peroxidase & $>2500$ & $>2500$ & 11.2 & 106.8 & 0.15 \\
\hline
\end{tabular}

ND: not determined; $a$ ) represents the ratio of $\mathrm{COX}-1 \mathrm{IC}_{50} / \mathrm{COX}-2 \mathrm{IC}_{50}$.

time-dependent inhibition of COX-1 and COX-2 (Fig. 2C).

Human Whole Blood Assay Since platelets do not express $\mathrm{COX}-2, \mathrm{TXB}_{2}$ produced by whole blood platelets was used to measure COX-1 inhibitory activity. Results showed that loxoprofen-SRS inhibited $\mathrm{TXB}_{2}$ production in a dosedependent manner. Similarly, loxoprofen-SRS also dose-dependently inhibited $\mathrm{PGE}_{2}$ production stimulated by LPS, which is used as a measure of COX-2 inhibitory activity. $\mathrm{IC}_{50}$ values for loxoprofen-SRS on COX-1 and COX-2 were $0.02 \mu \mathrm{M}$ and $0.28 \mu \mathrm{M}$, respectively (Fig. 3).

Rat Air Pouch Model of Carrageenan-Induced Inflammation Loxoprofen sodium showed a dose-dependent inhibition of $\mathrm{PGE}_{2}$ in the inflamed air pouch exudates. Platelet $\mathrm{TXB}_{2}$ and $\mathrm{PGE}_{2}$ production from the gastric mucosa were also dose-dependently inhibited by loxoprofen sodium. The $\mathrm{ED}_{50}$ values for air pouch $\mathrm{PGE}_{2}$, platelet $\mathrm{TXB}_{2}$ and gastric mucosa $\mathrm{PGE}_{2}$ inhibition were $2.0,0.34$, and $2.1 \mathrm{mg} / \mathrm{kg}$, respectively (Fig. 4).

Carrageenan-Induced Edema in Rat Hindpaws Loxoprofen sodium inhibited carrageenan-induced edema in a 


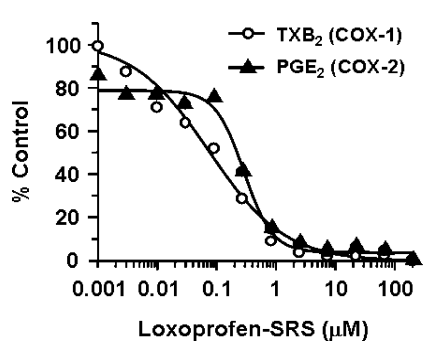

Fig. 3. Effect of Loxoprofen-SRS on $\mathrm{TXB}_{2}$ and $\mathrm{PGE}_{2}$ Production in Human Whole Blood

COX-1 inhibitory activity was determined by $\mathrm{TXB}_{2}$ production stimulated by calcium ionophore A-23187 $(n=5)$. COX-2 inhibitory activity was quantified by $\mathrm{PGE}_{2}$ production induced by LPS $(n=5)$.

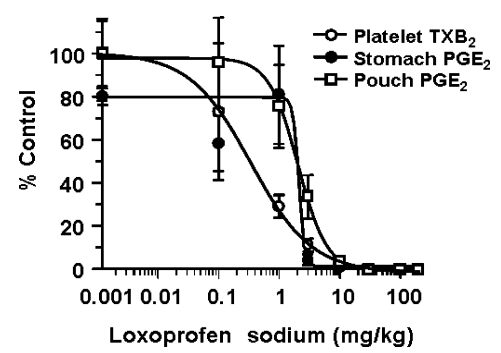

Fig. 4. Effect of Loxoprofen Sodium in the Rat Air Pouch Model of Inflammation

Loxoprofen sodium was administrated by oral gavage $1 \mathrm{~h}$ before initiating an inflammatory response in the air pouch with carrageenan. $\mathrm{PGE}_{2}$ present in the pouch exudates and extracted from the stomach mucosa and the $\mathrm{TXB}_{2}$ from the platelets were quantified by ELISA. Each point represents the percentage of control $\mathrm{PGE}_{2}$ or $\mathrm{TXB}_{2} \pm$ S.E.M. $(n=6)$.

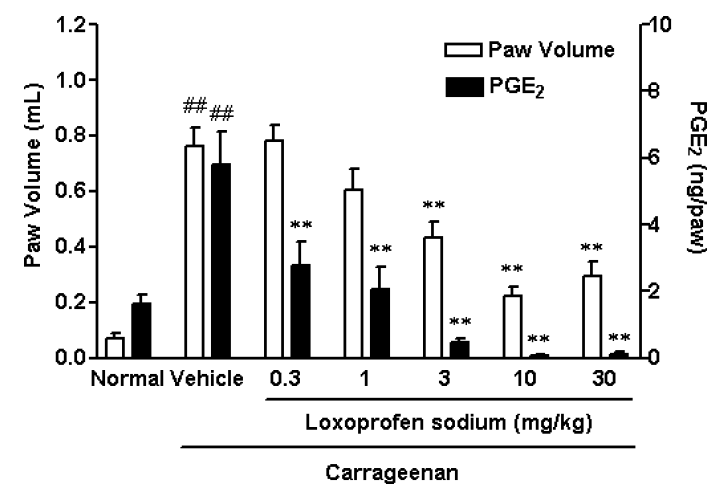

Fig. 5. Effect of Loxoprofen Sodium on Carrageenan-Induced Paw Edema and $\mathrm{PGE}_{2}$ Production in Rat Paw Tissue

Loxoprofen sodium was administered by oral gavage $2 \mathrm{~h}$ before carrageenan injection. Edema (open column) and $\mathrm{PGE}_{2}$ (closed column) were measured $3 \mathrm{~h}$ after carrageenan injection. Data for edema represent the mean of delta paw volume \pm S.E.M $(n=8)$. Data for $\mathrm{PGE}_{2}$ represent the mean \pm S.E.M. $(n=8) . \# p<0.01$, as compared with the normal control (Student's $t$-test ). $* * p<0.01$, as compared with the vehicle control (Dunnett's multiple range test).

dose-dependent manner with a maximal inhibition rate of $71 \%$. GGE $_{2}$ level in the paw exudates in the carrageenan-injected vehicle group was significantly higher than those from the normal group. Loxoprofen sodium dose-dependently inhibited these increases in $\mathrm{PGE}_{2}$ level (Fig. 5).

\section{DISCUSSION}

Loxoprofen sodium, a prodrug-type NSAID with relatively weak gatrointestinal ulcerogenicity, ${ }^{9)}$ is commonly used in Japan for the treatment of pain and inflammation. In the present study, we investigated the enzymologic and phar- macologic properties of loxoprofen sodium and its active metabolite with regard to inhibition of COX isozymes.

$\mathrm{IC}_{50}$ values of loxoprofen sodium and loxoprofen-SRS for COX-1 and COX-2 were determined using three different in vitro COX enzyme assays. It is well known that COX enzyme has two stages of catalytic activity. First, two oxygen molecules are incorporated into arachidonic acid to produce $\mathrm{PGG}_{2}$, followed by a peroxidase reaction that converts $\mathrm{PGG}_{2}$ to $\mathrm{PGH}_{2}$. Second, $\mathrm{PGH}_{2}$ is converted into biologically active products (e.g. $\mathrm{PGE}_{2}, \mathrm{PGI}_{2}$ or $\mathrm{TXA}_{2}$ ) by various individual synthase and reductase reactions. ${ }^{12}$ ) $\mathrm{Of}$ the three analysis methods, the chemical conversion of $\mathrm{PGH}_{2}$ to $\mathrm{PGE}_{2}$ provides an alternative method of measuring COX activity. The oxygen consumption assay relies on measurement of the rate of oxygen consumption in the reaction from arachidonic acid to $\mathrm{PGG}_{2}$, and the COX peroxidase activity can be assayed colorimetrically by monitoring the appearance of oxidized TMPD in the reaction from $\mathrm{PGG}_{2}$ to $\mathrm{PGH}_{2}$. In all three assays, loxoprofen sodium showed no apparent inhibition of COX-1 or COX-2, whereas loxoprofen-SRS inhibited both COX-1 and COX-2, though was apparent preferential potency for COX-1.

We also investigated the COX inhibitory activity of loxoprofen-SRS in human whole blood assay. This type of assay has been used as a pseudophysiological condition assay in the screening of compounds for their selectivity for COX-2 versus COX-1. ${ }^{13)}$ It is useful in that it may better reflect the in vivo effectiveness of NSAIDs in vitro, where it is subject to the effects of contributing factors such as protein binding. Loxoprofen-SRS inhibited both COX-1 and COX-2 though with 14-fold more potent activity against COX-1. These findings therefore confirm that loxoprofen-SRS will inhibit both COX-1 and COX-2 in the arachidonic acid metabolic pathways in human whole cells, but it tends to prefer COX-1.

In the present study of time-dependent mechanisms, loxoprofen-SRS showed time-dependent inhibition of COX-1 and COX-2. NSAIDs fall into two categories: those that inhibit COX reversibly (e.g. mefenamic acid) and those that demonstrate an irreversible time-dependent inhibition of activity in vitro (e.g. indomethacin). ${ }^{14,15)}$ Rome and Lands demonstrated that some NSAIDs with carboxylic acid moieties at certain positions displayed time-dependent inhibition of COX. ${ }^{14)}$ Since loxoprofen-SRS showed time-dependent inhibition of COX-1 and COX-2, and has carboxylic moiety in its structure, it inhibits COX-1 and COX-2 in an irreversible time-dependent manner. Celecoxib, a specific COX-2 inhibitor, is known to inhibit COX-2 in a slow, time-dependent process and to be a weak competitive inhibitor of COX-1. ${ }^{10)}$ These findings suggest that the mode of loxoprofen-SRS's inhibition of COX-2 is similar to that of COX-2 inhibitors, whereas that for COX-1 is different from that of COX-2 inhibitors.

Oral administration of loxoprofen sodium dose-dependently decreased the $\mathrm{PGE}_{2}$ content of pouch exudates and stomach $\mathrm{PGE}_{2}$ in a rat carrageenan-induced air pouch model. This model had been widely used to assess a compound's inhibitory potency against inflammatory and physiological PG production. ${ }^{16)}$ Masferrer et al. showed that the lining of the carrageenan-challenged air pouch contains a significant level of COX-2 and a steady-state level of COX-1.6) These findings imply that the majority of the $\mathrm{PGE}_{2}$ in the pouch was derived from COX-2, whereas $\mathrm{PGE}_{2}$ in the stomach was de- 
rived from COX-1. In the present study, loxoprofen sodium inhibited the $\mathrm{PGE}_{2}$ content of both pouch exudates and stomach. $E_{50}$ values of these effects were very similar (pouch exudate $\mathrm{PGE}_{2}, 2.0 \mathrm{mg} / \mathrm{kg}$; stomach $\mathrm{PGE}_{2}, 2.1 \mathrm{mg} / \mathrm{kg}$ ), indicating that loxoprofen sodium inhibits both COX isozymes in vivo within the same dose range. In vivo inhibitory potency for COX-1 and COX-2 was also suggested by the results of carrageenan-induced footpad edema (COX-2) and platelet $\mathrm{TXB}_{2}(\mathrm{COX}-1)$ in rat air pouch model. However, in vitro study, loxoprofen-SRS inhibited COX-1 more potently than COX-2. In the air pouch model study, $\mathrm{ED}_{50}$ value for the pouch exudate and the platelet $\mathrm{TXB}_{2}$ were $2.0 \mathrm{mg} / \mathrm{kg}$ and $0.34 \mathrm{mg} / \mathrm{kg}$, respectively. The differences in the $\mathrm{ED}_{50}$ values of the pouch exudate and the platelet $\mathrm{TXB}_{2}$ were considered to be the difference in the tissue concentration of loxoprofenSRS. Sugimoto et al. reported that the plasma concentration of loxoprofen-SRS after oral administration of loxoprofen sodium was higher than the inflammatory exudates in a carrageenan induced rat paw edema model. ${ }^{8)}$ As a result of the differences in the drug concentration, there is a possibility that the $\mathrm{ED}_{50}$ value for platelet $\mathrm{TXB}_{2}$ was lower than the pouch exudate $\mathrm{PGE}_{2}$. Overall, loxoprofen sodium activity is attributable to its active metabolite, loxoprofen-SRS, and that loxoprofen-SRS shows non-selective inhibition for COX.

Although some differences were seen in the inhibitory potency for COX isozymes, we found no distinction between the loxoprofen sodium and traditional NSAIDs inhibitory profiles against COX isozymes either in vitro or in vivo.

Loxoprofen sodium is transformed into the functionally active form via aldehyde dehydrogenase only after absorption in the gastrointestinal tract. Therefore, in the clinical setting, loxoprofen sodium is less irritable and induces a lower degree of injury to the digestive tract than traditional NSAIDs. In a small-scale clinical experiment, Kawano et al. showed that a single topical application of loxoprofen sodium is safer than indomethacin. ${ }^{17)}$ However, it is difficult to judge the gastrointestinal safety of loxoprofen by a single topical application in a small-scale study. Rather, based on a recent clinical study of COX-2-selective inhibitors in which endoscopic examination showed that these compounds are similar to placebo in their effects on the gastroduodenal mu- cosa, ${ }^{18,19)}$ we anticipate that the safety of loxoprofen sodium would be more rigorously determined by large-scale clinical endoscopic studies.

\section{REFERENCES}

1) Smith W. L., Marnett L. J., Biochem. Biophys. Acta, 1083, 1-17 (1991).

2) Smith W. L., Biochem. J., 259, 315-324 (1989).

3) DuBois R. N., Abramson S. B., Crofford L., Gupta R. A., Simon L. S., Van de Putte L. B. A., Lipsky P. E., FASEB J., 12, 1063-1073 (1998).

4) Vane J. R., Bakhle Y. S., Botting R. M., Annu. Rev. Pharmacol. Toxicol., 38, 97-120 (1998).

5) Gierse J. K., Hauser S. D., Creely D. P., Koboldt C., Rangwala S. H., Isakson P. C., Seibert K., Biochem. J., 305, 479—484 (1995).

6) Masferrer J. L., Zweifel B. S., Manning P. T., Hauser S. D., Leahy K. M., Smith W. G., Isakson P. C., Seibert K., Proc. Natl. Acad. Sci. U.S.A., 91, 3228-3232 (1994).

7) Carson J. L., Willett L. R., Drugs, 46 (Suppl. 1), 243-248 (1993).

8) Sugimoto M., Kojima T., Asami M., Iizuka Y., Matsuda K., Biochem. Pharmacol., 42, 2363-2368 (1991).

9) Misaka E., Yamaguchi T., Iizuka Y., Kamoshida K., Kojima T., Kobayashi K., Endo Y., Misawa Y., Kobayashi S., Tanaka K., Pharmacometircs, 21, 753-771 (1981).

10) Gierse J. K., Koboldt C. M., Walker M. C., Seibert K., Isakson P. C., Biochem. J., 339, 607-614 (1999).

11) Walker M. C., Kurumbail R. G., Kiefer J. R., Moreland K. T., Koboldt C. M., Isakson P. C., Seibert K., Gierse J. K., Biochem. J., 357, 709718 (2001).

12) Smith W. L., Garavito R. M., DeWitt D. L., J. Biol. Chem., 271, 33157-33160 (1996).

13) Patrignani P., Panara M. R., Greco A., Fusco O., Natoli C., Iacobelli S., Cipollone F., Ganci A., Creminon C., Maclouf J., Patrono C., J. Pharmacol. Exp. Ther, 271, 1705-1712 (1994).

14) Rome L. H., Lands W. E., Proc. Natl. Acad. Sci. U.S.A., 72, 48634865 (1975).

15) Kulmacz R. J., Lands W. E., J. Biol. Chem., 260, 12572-12578 (1985).

16) Sedgwick A. D., Lees P., Agents Actions, 18, 429-438 (1986).

17) Kawano S., Tsuji S., Hayashi N., Takei Y., Nagano K., Fusamoto H., Kamada T., J. Gastroenterol. Hepatol., 10, 81-85 (1995).

18) Simon L. S., Weaver A. L., Graham D. Y., Kivitz A. J., Lipsky P. E., Hubbard R. C., Isakson P. C., Verburg K. M., Yu S. S., Zhao W. W., Geis G. S., JAMA, 282, 1921-1928 (1999).

19) Emery P., Zeidler H., Kvien T. K., Guslandi M., Naudin R., Stead H., Verburg K. M., Isakson P. C., Hubbard R. C., Geis G. S., Lancet, 354, 2106-2111 (1999). 